\section{Identificar-se com os outros consumidores minimiza o efeito crowding? O papel da similaridade percebida}

\author{
Edvan Cruz Aguiar e Salomão Alencar de Farias
}

\section{RESUMO}

O estudo propõe que a identificação do consumidor com os outros clientes que compartilham o mesmo ambiente de loja minimiza o efeito crowding. Assim, investigou-se de que modo a similaridade percebida modera a direção dos efeitos da densidade humana sobre a percepção de crowding, bem como da percepção de crowding sobre a satisfação do consumidor. A partir de um estudo de desenho experimental, hipóteses de pesquisa foram testadas, e os achados evidenciam que a similaridade percebida contribui para que o efeito negativo do crowding seja mitigado. Os resultados também sugerem que esta variável se relaciona com a atitude do consumidor com o varejista, com os produtos e com a própria experiência de compra. O trabalho introduz uma variável até então negligenciada pelas pesquisas envolvendo o fenômeno crowding no varejo, ampliando as possibilidades de pesquisa sobre o papel da dimensão social do ambiente varejista sobre o comportamento do consumidor.

Palavras-chave: Similaridade Percebida; Crowding; Varejo.

Does the identification with other consumers minimize the crowding effect? The perceived similarity's role

\section{ABSTRACT}

The study proposes that the consumer's identification towards other clients who share the same retail environment minimizes the crowding effect. Thus, it was investigated how perceived similarity moderates the effect of human density on crowding perception, as well as crowding perception on consumer satisfaction. From an experimental study design, research hypotheses have been tested, and findings show that perceived similarity contributes to the negative effect of crowding being mitigated. The results also suggest that this variable is related to the consumer's attitudes towards retailers, products and shopping experience. This work introduces a variable that has been neglected, regarding the crowding phenomena in the retail environment. It also extends the research possibilities on the role of social dimension of the retailing environment over the consumer behavior.

Keywords: Perceived Similarity; Crowding; Retailing.

Recebido em: 04/07/2018 Revisado em: 14/08/2018 Aprovado em: 01/09/2018

Check for updates

Edvan Cruz Aguiar (D), Universidade Federal de Campina Grande, Brasil Doutor em Administração de Empresas, UFPE, Brasil

edvan.aguiar@ufcg.edu.br

Salomão Alencar de Farias (iD,

Universidade Federal de Pernambuco, Brasil

Doutor em Administração de Empresas, USP, Brasil

saf@ufpe.br 


\section{Introdução}

No que se refere à multiplicidade de elementos constitutivos de ambientes varejistas capazes de influenciar o comportamento do consumidor, ressalta-se a dimensão social (Aguiar \& Farias, 2015). A relevância dos 'outros' clientes se sobressai, uma vez que eles tendem a influenciar respostas emocionais, determinando, em grande medida, atitudes e comportamentos de compra (Mcgrath \& Otnes, 1995; Evans, Christiansen, \& Gill, 1996; Martin, 1996; Grove \& Fisk, 1997).

Os fatores sociais, sobretudo quando se trata da presença de outros consumidores, apresentam-se como aspectos importantes do ambiente varejista, especialmente ao estudar a sua relação com o comportamento do consumidor. Destarte, o fenômeno conhecido por crowding (ou aglomeração) (Stokols, 1972; Eroglu \& Harrel, 1986; Hui \& Bateson, 1991) refere-se a um dos fatores sociais que tem recebido a atenção de pesquisadores nos últimos anos, sobretudo no contexto brasileiro (Brandão, Parente, \& Oliveira, 2010; Aguiar, Farias, Gomes, \& Santos, 2015; Quezado, Costa, Peñaloza, \& Matos, 2016).

Os primeiros estudos e discussões acadêmicas sobre o fenômeno crowding, sob a ótica do consumidor em ambientes varejistas, datam do início da década de 1970 (Loo, 1973; Harrell \& Hutt, 1976). Tais estudos procuraram entender como os consumidores reagem aos espaços com diferentes níveis de densidade humana. Inicialmente, a densidade humana foi considerada um elemento desencadeador de emoções e experiências de compra negativas. Não obstante, ao longo das últimas décadas, surgiram evidências de que ela não constitui, necessariamente, um aspecto negativo, pois é capaz inclusive de criar estados de satisfação no consumidor em certos contextos de compra (Machleit, Eroglu, \& Mantel, 2000).

Tais divergências revelam a possibilidade de haver variáveis que influenciam a relação entre a percepção de crowding e as respostas dos consumidores. Assim, a despeito da quantidade de estudos acerca desse fenômeno, verifica-se que ainda são necessárias investigações que explorem outros fatores sociais capazes de influenciar as respostas dos consumidores. O raciocínio aqui apresentado sustenta que percepções e atitudes individuais são constantemente moldados por fatores sociais e contextuais, além da densidade humana.

Neste sentido, argumenta-se que a similaridade percebida em geral prediz preferências individuais em termos de distância para com as demais pessoas que compartilham o mesmo ambiente, de modo que pode assumir um papel moderador ao investigar a dimensão social de ambientes varejistas. Ela é definida como o grau com que os outros clientes são percebidos como semelhantes ao consumidor que os observa (Brocato, Voorhees, \& Baker, 2012).

Credita-se, assim, relevância à similaridade percebida, ao considerar sua capacidade de explicar o papel da proximidade física no estudo da relação entre ambientes varejistas e o comportamento do consumidor. Partindo-se do pressuposto de que a proximidade física entre consumidores pode aumentar ou diminuir preferências individuais por determinados produtos, $\mathrm{Xu}$, Shen 
e Wyer (2012) constataram que essa pista social resulta em desconforto quanto à experiência do consumidor.

Kwon, Ha e Im (2016) analisaram a influência da similaridade percebida na satisfação para com a compra e, além de ressaltar o papel que a presença social exerce no ambiente varejista, sobretudo no comportamento individual, evidenciaram um elo positivo entre essa variável e a experiência de compra. Esses achados sugerem que essa variável também interfere no efeito que o crowding exerce sobre as repostas consumidor.

Mesmo com as pesquisas recentes acerca da dimensão social do ambiente varejista, acredita-se que o entendimento do papel da similaridade percebida no estudo do fenômeno crowding pode enriquecer o corpo teórico de conhecimento na área. Entende-se que os efeitos da densidade humana sobre percepção de crowding, e desta na satisfação do consumidor, pode mudar de acordo com outras variáveis, conforme sugerido por Brandão e Parente (2012). Desse modo, compreender a influência da similaridade percebida mostra-se pertinente, uma vez que ela pode ajudar a melhor explicar a direção do impacto da densidade humana sobre o comportamento do consumidor.

Diante do exposto, o presente artigo se propôs a investigar de que modo a similaridade percebida modera a direção dos efeitos da densidade humana sobre a percepção de crowding, bem como da percepção de crowding sobre a satisfação do consumidor, em ambiente varejista com diferentes níveis de densidade humana, proximidade física e perfil dos outros consumidores.

É importante mencionar que, a despeito da crescente atenção atribuída à influência da presença social sobre o comportamento do consumidor, seu efeito não tem sido examinado quando se analisa o fenômeno crowding, especificamente quanto ao papel da similaridade percebida. Portanto, esta pesquisa mostra-se útil ao passo em que fornece evidências que ajudam a melhor compreender a dinâmica da dimensão social do ambiente varejista no comportamento do consumidor.

\section{Revisão Teórica}

\section{Crowding no Varejo}

No varejo, o fenômeno crowding tem sido bastante útil para compreensão da maneira pela qual os sujeitos são impactados por situações em que lojas se encontram cheias de outros clientes e/ou produtos. Os primeiros trabalhos no contexto varejista foram realizados nas décadas de 1970 e 1980, por pesquisadores como Harrell, Hutt, Anderson, Eroglu e Machleit. A experiência de crowding corresponde ao resultado de uma interação entre aspectos físicos, sociais e pessoais. Assume-se que, mesmo em condições nas quais o ambiente varejista apresente altos níveis de densidade humana, um consumidor, com elevado grau de controle percebido, tenderá a responder menos negativa à densidade (White, 1959). 
Entende-se que a percepção do consumidor com a densidade humana está atrelada às suas expectativas e/ou motivações de compra, às experiências anteriores e até traços de personalidade (Michon, Chebat, \& Turley, 2005). Neste sentido, argumenta-se que a identificação do cliente com os demais consumidores exerce um papel importante na forma como a presença social é percebida. De acordo com Yüksel (2009), a percepção de crowding não ocorre isoladamente, de sorte que é pertinente considerar as demais pistas ambientais capazes de explicar tal fenômeno.

Para que a densidade humana exerça efeito na percepção de crowding, fatores sociais e pessoais precisam contribuir para que o indivíduo sinta que seu espaço físico foi restrito (Rüstemli, 1991). Isso sugere que a direção dos efeitos do crowding pode variar conforme a presença de outros fatores capazes de mitigar ou ampliar seu impacto no comportamento do consumidor. Respostas negativas ocorrem em situações nas quais o ambiente físico encontra-se com alta densidade humana. No entanto, a proximidade física entre as pessoas que compartilham o espaço também pode exercer influência na maneira como o crowding é percebido.

Assim, um ambientevarejista, ainda que com níveis elevados de densidade humana, pode ser avaliado positivamente pelo consumidor na medida que a presença dos outros clientes seja considerada agradável. Situações em que o consumidor divide o ambiente com amigos e/ou familiares, ou até mesmo desconhecidos que compartilham uma identidade social, podem caracterizar contextos em que a proximidade física e a própria densidade humana não causariam respostas negativas, mas o oposto disso.

A presença dos outros clientes estimula motivos sociais relacionados à compra, enquanto uma loja quase vazia levanta dúvidas acerca do produto e da qualidade dos serviços (Pan \& Siemens, 2011; Uhrich \& Luck, 2012). Consumidores podem perceber a presença dos outros em seu espaço social (ambiente varejista) como aceitável e, consequentemente, responderem favoravelmente, ainda que estejam em situações de alta densidade humana. Contudo, a aglomeração humana por si só não é capaz de explicar percepções, atitudes e comportamentos individuais. Ou seja, outras pistas sociais também precisam ser consideradas.

Maeng, Tanner e Soman (2013), ao conduzirem seis estudos com o intuito de responder se a mera densidade humana presente no ambiente de loja afetaria as escolhas e preferências individuais, constataram que os efeitos do crowding são atenuados quando a presença social é composta por membros do grupo. Assim, considerando que, de um modo geral, os membros de um determinado grupo que compartilham uma mesma identidade social (ainda que desconhecidos) são similares numa variedade de características, espera-se que a similaridade percebida exerça um efeito moderador na relação entre percepção de crowding e as repostas do consumidor.

Muito embora seja reconhecida a influência do crowding no comportamento de compra do consumidor no ambiente varejista, percebe-se que variáveis como a similaridade percebida ajudam a apreender a maneira pela qual lojas cheias não causam, necessariamente, respostas negativas, sugerindo um papel moderador desta variável. 


\section{Similaridade Percebida com os outros Consumidores}

Um aspecto importante que deve ser considerado ao se investigar o fenômeno crowding, sobretudo o seu impacto sobre as respostas individuais, é verificar se a presença de outras pessoas facilita ou inibe motivações e objetivos pessoais em ambientes físicos. Neste sentido, as experiências de crowding também podem ser atribuídas à violação da distância interpessoal, ou seja, da proximidade física entre os indivíduos.

A influência que o fator social exerce individualmente sobre o consumidor é considerada fundamental para o entendimento das suas respostas em termos de comportamento de consumo (Ryan, 1982). Em se tratando de ambientes varejistas, as interações e demais pistas sociais (ex.: número de pessoas, aparência em geral e comportamento) acabam assumindo papel determinante na experiência de compra.

A Teoria do Impacto Social (Latané, 1981) e o Paradigma Similaridade - Atração (Byrne, 1971) correspondem às abordagens teóricas advindas da psicologia social, úteis ao estudo do comportamento do consumidor, sobretudo quando se investiga o papel da dimensão social dos ambientes varejistas. Essas duas abordagens suportam o argumento central desta investigação, ao ajudarem a compreender os mecanismos envolvidos no estudo do fenômeno crowding, especialmente quando a similaridade percebida é considerada.

O impacto potencial de outros consumidores pode ser explicado pela Teoria do Impacto Social (Latané, 1981), a qual sugere que pessoas exercem influência emocional e comportamental sobre outras. Essa teoria objetiva distinguir as maneiras pelas quais um indivíduo afeta outro em função de aspectos como a restrição de tempo e espaço e, sobretudo, como o impacto sobre as pessoas é moderado pela importância, pela proximidade física e pelo número de indivíduos em um dado ambiente.

O impacto social é definido como qualquer influência sobre os sentimentos, pensamentos e comportamentos individuais, exercida por uma presença real, insinuada ou imaginada, ou por ações de outros indivíduos (Latané, 1981). Ao revisar a literatura atinente ao fenômeno crowding, pouco se conhece acerca de registros empíricos que investigaram o efeito interativo de pistas sociais como a densidade humana e a proximidade física sobre as respostas do consumidor.

Ao trazer os princípios da Teoria do Impacto Social para o estudo do comportamento do consumidor, entende-se que é possível ampliar a compreensão acerca das razões que determinam preferências, avaliações e comportamentos individuais, em função das pistas sociais. Mais especificamente, essa teoria contribui para compreender o papel de pistas sociais como a densidade humana e a distância interpessoal.

O número de forças sociais corresponde à quantidade de pessoas presentes no mesmo ambiente físico. Assim, pode-se assumir que a densidade humana diz respeito à pista social preconizada pela Teoria do Impacto Social (LATANÉ, 1981). Os resultados do estudo conduzido por Bearden e Etzel (1982), ao investigar como grupos de referência influenciam decisões individuais acerca de marcas e produtos, também poderiam ser explicados à luz da SIT (Social Impact Theory). 
O Paradigma Similaridade - Atração (Byrne, 1971) também é adequado à compreensão dos traços observáveis dos consumidores que compartilham um mesmo ambiente varejista, capazes de influenciar percepções e atitudes. De acordo com essa abordagem, as pessoas são mais propensas a serem atraídas por coisas semelhantes (ex.: pessoas e objetos), em função de aspectos como semelhança e aparência físicas.

Utilizando os conceitos de disssonância cognitiva e condicionamento clássico, Byrne (1971) argumenta que atitudes similares servem como reforços positivos. Por exemplo, pessoas que compartilham ideias e opiniões validam atitudes umas das outras e, consequentemente, reforçam crenças e até comportamentos. O efeito da similaridade também ocorre a partir do processamento de informação, ou seja, um sujeito utiliza as informações referentes à(s) outra(s) para formar suas impressões (Byrne; 1971; Grush, Clore, \& Costin, 1975; Blau \& Katerberg, 1982).

Argumenta-se que os indivíduos fazem uso de características próprias como referência para acessarem os atributos de terceiros e inferir sobre eles. Desse modo, o alinhamento entre as informações compartilhadas (ex.: idade, aparência, comportamento, atitudes e personalidade) gera respostas individuais positivas ou negativas, caso existam (in)congruências na perspectiva de quem observa a presença social. No caso desta pesquisa, trata-se do consumidor ao observar os demais clientes que compartilham o mesmo ambiente de loja.

O trabalho de McGrath e Otnes (1995) não só evidenciou o poder dos contextos físico e social da loja, como também sugeriu o papel da similaridade percebida do consumidor. Para os pesquisadores, os clientes tendem a interagir com aqueles visualmente julgados como semelhantes, e tal homogeneidade é percebida ao considerar pistais sociais de fácil observação, como idade, gênero e aparência (vestuário).

A literatura na área reconhece que consumidores, sendo parte do ambiente, podem afetar a experiência de compra (Baker, 1986; Martin \& Pranter, 1989; Bitner, 1992; Martin, 1996; Baker, Parasuraman, \& Grewal, 2002; Moore, Moore, \& Capella, 2005). A percepção do consumidor em relação aos outros envolve diversas pistas sociais, e estas correspondem a estímulos que influenciam respostas individuais.

Kim e Lee (2012) afirmam que os outros consumidores correspondem a um fator multidimensional composto pelas seguintes pistas sociais: número de clientes, gênero, aparência, traje e comportamento. Neste sentido, a similaridade percebida é formada a partir da observação de pistas sociais, como o perfil dos outros clientes. Em outras palavras, o consumidor forma suas impressões acerca da presença social.

A partir da identificação de lacuna teórica concernente à ausência de uma escala global útil à avaliação das percepções que o indivíduo tem dos outros consumidores durante experiências de consumo, Brocato et al. (2012) estabeleceram três dimensões do conceito, que os próprios autores denominaram de Other Customer Perception (Percepção dos Outros Consumidores - OCP), e desenvolveram uma escala com o propósito de mensurar esse construto. Dentre as dimensões da escala, o construto 
similaridade percebida foi delineado e validado. Ademais, os achados evidenciaram que esta variável efetivamente se relaciona com atitudes e comportamentos do consumidor.

Aguiar e Farias (2015), ao aplicarem a escala OCP no contexto brasileiro, encontraram um efeito significativo da presença de outros consumidores sobre respostas individuais durante o processo de compra (emoções e valor hedônico derivado da compra). Assim, reitera-se o argumento de que ambientes frequentados por consumidores "similares" conduzem a experiências mais prazerosas, com intenções de comportamento mais favoráveis.

Pesquisadores têm indicado que o ponto mais crítico para a previsão de atração interpessoal está no fato das pessoas acreditarem que as outras são semelhantes, ainda que não sejam parecidas efetivamente (Montoya, Horton, \& Kirchner, 2008; Montoya \& Horton, 2012). Afirma-se não ser possível controlar a maneira como as pistas sociais (idade, aparência, vestuário) são analisadas pelo consumidor, já que duas pessoas podem interpretar um mesmo atributo (característica) distintamente. Contudo, é coerente sugerir que respostas favoráveis são mais propensas a ocorrerem quando há percepção de similaridade e vice versa.

\section{Hipóteses de Pesquisa}

Ao discutir a influência da dimensão social no comportamento do consumidor, a Teoria do Impacto Social sugere que as forças sociais (quantidade de pessoas, a proximidade física e o grau de importância atribuída à presença social) influenciam respostas individuais de cunho afetivo, cognitivo e comportamental.

Violações a normas de espaço pessoal podem produzir reações negativas por parte da pessoa que percebe seu lugar invadido. Algumas dessas respostas podem corresponder a pequenas mudanças de comportamento, interposição de barreiras reais ou simbólicas, ou até mesmo reações de caráter verbal (Harris, Luginbuhl, \& Fishbein, 1978).

Quando uma presença social, em estreita proximidade, é numerosa em quantidade, o espaço pessoal do indivíduo é invadido, criando assim estresse e desconforto. Contudo, quando a presença social se encontra mais distante, tal espaço não é invadido, independentemente do tamanho da presença social (Kraut, 1982). Com base no exposto, formulou-se as seguinte hipótese:

H1: Consumidores distantes fisicamente da presença social no ambiente varejista percebem menos crowding em comparação com aqueles fisicamente próximos.

Apesar da densidade contribuir para a ocorrência de comportamentos verbais negativos (ex.: reclamação), situações de crowding não resultam, necessariamente, em experiência estressante, posto que a atração entre os indivíduos presentes em um dado ambiente assume um caráter interveniente nas respostas inerentes às situações em que o crowding é percebido (Emiley, 1975). 
A similaridade percebida prediz atração interpessoal, e a crença de que os outros são semelhantes contribui para que essa relação ocorra (Byrne, 1971; Montoya et al., 2008). A presença dos outros consumidores pode influenciar o tempo de permanência do cliente em ambientes de consumo (Tombs \& Mccoll-Kennedy, 2010). Ademais, a similaridade percebida possui impacto positivo nas intenções de comportamento de aproximação e impacto negativo nas intenções de afastamento (Brocato et al., 2012).

Desta forma, é sugerido que o efeito da densidade humana pode ser atenuado quando a presença social é composta por membros do grupo (Maeng et al., 2013; Alnabulsi \& Drury, 2014).

H2a: A similaridade percebida modera o impacto da densidade humana sobre a percepção de crowding.

As pistas sociais do espaço varejista contribuem para que o consumidor estabeleça julgamentos quanto à sua experiência de consumo. De maneira geral, tem-se assumido que a percepção de crowding leva o consumidor a estabelecer atitudes negativas. Entretanto, é reconhecido que variáveis moderadoras podem influenciar esta relação (Lee, Kim, \& Li, 2011; Pan \& Siemens, 2011; Machleit, Eroglu, \& Mantel, 2000; Brandão \& Parente, 2012; Mehta, Sharma, \& Swami, 2013).

Assim sendo, espera-se que o efeito negativo da percepção de crowding sobre as atitudes do consumidor (satisfação) seja mitigado pelo impacto da similaridade percebida nesta relação.

H2b: O impacto negativo da Percepção de Crowding sobre a Satisfação do consumidor será menor quando este se identificar com a presença social no ambiente varejista.

\section{- Procedimentos Metodológicos}

A investigação assume natureza experimental, cujo principal propósito foi obter evidências relativas às relações de causa e efeito entre variáveis (Campbell \& Stanley, 1979; Hernandez, Basso, \& Brandão, 2014). Assim, foi delineado um experimento do tipo fatorial (between subjects), em que cada um dos grupos de respondentes foi submetido a apenas uma condição. Esse tipo de delineamento é comum nos estudos em marketing e comportamento do consumidor (Borges, Chebat, \& Babin, 2010; Jin, He, \& Zhang, 2014; Mcferran \& Argo, 2014).

A relação de causa e efeito deste estudo tem como fatores: a densidade humana (crowding, em sua perspectiva objetiva); a proximidade física (distância interpessoal); e o perfil dos outros consumidores. Portanto, trata-se de um experimento de desenho fatorial (between subject) 2 (densidade humana) $X 2$ (proximidade física) $\times 2$ (perfil dos outros consumidores).

Foi aplicada a técnica de dados intergrupo (Hair, Anderson, Tatham, \& Black, 2005; Malhotra, 2006), em que cada sujeito da pesquisa é exposto a apenas uma situação do experimento. Tal medida se justifica pelo fato do membro ter sua resposta influenciada em função da situação anterior, podendo gerar um viés nas suas impressões quanto às combinações da fatorial. 
Delineamento dos cenários do (experimento)

Uma loja de bens (vestuário) foi escolhida para que as condições do experimento fossem estabelecidas. Para tanto, fez-se uso de observações não estruturadas e não participante (Flick, 2009). Para o delineamento dos cenários, figurantes foram convidados a participar da construção do contexto de compra na loja, por meio de uma sessão fotográfica.

A ideia foi reproduzir as situações a partir da combinação dos três fatores e seus respectivos níveis. Um profissional de fotografia foi contratado para conduzir a sessão, orientar os figurantes e também editar as imagens que representassem as células da fatorial. Constatou-se que seriam necessários, no mínimo, 10 modelos, entre homens e mulheres, para se chegar ao resultado esperado, segundo as duas situações de crowding (baixa e alta).

Para o fator proximidade física, durante a sessão de fotos, a câmera ficou posicionada em dois locais diferentes a fim de obter o efeito almejado, obedecendo às seguintes medidas (distâncias): $60 \mathrm{~cm}$ na condição próxima fisicamente dos outros consumidores e $2 \mathrm{~m}$ e $50 \mathrm{~cm}$ para a condição distante fisicamente dos outros clientes. Tal critério foi baseado no trabalho de (Argo, Dahl, \& Manchanda, 2005). Além disso, a descrição do cenário e do contexto de compra, presente no instrumento de coleta, reforçou a manipulação dessa variável, conforme feito em estudos anteriores (Miao, Mattila, \& Mount, 2011; Wu, Mattila, \& Han, 2014).

Com relação ao perfil dos outros consumidores, a indumentária correspondeu à pista social escolhida pelo pesquisador para ser inferida pelos sujeitos da pesquisa enquanto antecedente da percepção de similaridade. A partir das observações realizadas antes da escolha da loja, foram definidos dois perfis distintos, considerando apenas seu traje enquanto pista social.

Para a escolha das fotografias (condições do experimento) a serem utilizadas no estudo, especialistas na área de varejo e comportamento do consumidor foram convidados a analisar as imagens. Ressalte-se que a utilização de vídeos e fotografias tem sido recorrente em experimentos na área de marketing e comportamento do consumidor (Brandão, 2011; Maeng et al., 2013; Zhang, Burke, \& Leykin, 2014).

\section{Procedimentos de coleta dos dados}

A amostra foi não probabilística e por conveniência, tendo como critério de escolha a acessibilidade e a disponibilidade dos sujeitos de pesquisa em colaborar com o estudo. Os respondentes corresponderam a estudantes de graduação e pós-graduação, das mais diversas áreas, em instituições de ensino superior (públicas ou privadas). O instrumento de coleta foi estruturado em três partes. A primeira, com questões referentes aos construtos teóricos (similaridade percebida, percepção de crowding e satisfação). A segunda apresentou perguntas de validação das manipulações do cenário da pesquisa. E, por fim, foram demonstradas questões de caracterização da amostra.

Os construtos de interesse da pesquisa foram do tipo Likert de 7 pontos, variando de (1) discordo totalmente a (7) concordo totalmente. É importante comentar que antes da coleta dos dados, as escalas foram traduzidas da 
língua inglesa para o português e, posteriormente, fez-se o inverso, a fim de identificar possíveis discrepâncias no uso de palavras e garantir fidedignidade às perguntas originais (back translation). Esse processo foi realizado com o auxílio de profissional especialista em tradução, nativo em inglês e com experiência de mais de 10 anos nesse tipo de trabalho.

Para o questionário, também foi criado um cenário de compra para que o sujeito de pesquisa pudesse se sentir imerso às situações do experimento, mais especificamente, às condições de densidade humana, proximidade física e perfil dos outros consumidores. Antes da sua aplicação (incluindo a descrição do contexto de compra e a fotografia representativa do cenário) o pesquisador explicou rapidamente o propósito da pesquisa e fez as recomendações pertinentes no que diz respeito ao correto preenchimento do documento.

Para condução da efetiva coleta de dados, junto com o questionário e a descrição do contexto de compra, as fotografias (os cenários) foram entregues para cada sujeito de pesquisa correspondente a cada grupo/ célula da fatorial, de forma aleatória. A aplicação ocorreu em sala de aula, participando apenas os sujeitos que espontaneamente se inclinaram a colaborar com a pesquisa.

As sessões de coleta duraram em média 25 minutos, considerando o tempo para apresentar a proposta do estudo aos sujeitos de pesquisa, passar orientações gerais sobre o preenchimento dos questionários a partir da descrição do cenário e análise da imagem (fotografia) e recolher os instrumentos de coleta. Antes da entrega dos questionários (incluindo descrição do contexto de compra e fotografia representativa do cenário), o pesquisador explicou rapidamente sobre o propósito da pesquisa e fez as recomendações pertinentes no que diz respeito ao correto preenchimento do documento.

\section{Procedimentos de análise dos dados}

Antes da análise propriamente dita, a inspeção na base de dados (também conhecidos como missing data) e a verificação de valores excessivamente reduzidos e elevados (denominados outliers) foram feitas. Testes de normalidade das distribuições e de validação das escalas (coeficiente alfa de Cronbach e Análise Fatorial Exploratória - AFE), foram aplicados (Cronbach, 1951; Aaker, Kumar, \& Day, 2001, 2001; Hair et al., 2005; Malhotra, 2006). A confiabilidade composta e a variância média extraída também foram verificadas.

A análise propriamente dita envolveu estatísticas descritivas e inferenciais. A caracterização da amostra, a verificação de sua distribuição, considerando os cenários do experimento, bem como a observância de padrões de respostas referentes aos construtos, corresponderam às análises preliminares. Adicionalmente, empregaram-se técnicas multivariadas para atender aos propósitos da pesquisa, notadamente a análise de moderação (Preacher, Rucker, \& Hayes, 2007; Preacher \& Hayes, 2008; Vieira, 2009; Hayes, 2013). 
A análise de moderação utilizada nesta pesquisa partiu da proposta de Baron e Kenny (1986), complementada pelas contribuições de Hayes (2013), que se fundamentam em modelos de regressão linear tradicionais (método dos mínimos quadrados). Foi utilizado um macro (script computacional) livremente disponível para o software SPSS, chamado PROCESS.

\section{Análise e Discussão dos Resultados}

Ao final do processo de depuração da matriz, a base de dados contemplou 358 casos válidos, dos quais $57 \%$ são do sexo feminino e $43 \%$ do sexo masculino. A média de idade dos participantes foi de 23 anos. Com relação à Análise Fatorial Exploratória, o método de rotação ortogonal das variáveis permitiu a identificação dos fatores (variáveis latentes). O KMO foi de 0,952 e o Teste de Esfericidade de Bartlett foi de 19530,975, com 1176 graus de liberdade a um nível de significância de 0,001. Tais resultados confirmaram a estrutura dos fatores (Tabachnick \& Fidell, 2013).

Para avaliar a adequação dos construtos teóricos, o pesquisador também fez uso das análises de validade convergente e discriminante, conforme sugerido por Fornell e Lacker (1981). Assim, foram conferidas a confiabilidade das escalas (simples e composta) e a variância média extraída.

Tabela 1. Cronbach's Alpha, Confiabilidade Composta e Variância Média

\begin{tabular}{lccc}
\hline \multicolumn{1}{c}{ Construto } & $\begin{array}{c}\text { Cronbach's Alpha } \\
\text { (Coeficiente) }\end{array}$ & $\begin{array}{c}\text { Confiabilidade } \\
\text { Composta }\end{array}$ & $\begin{array}{c}\text { Variância Média } \\
\text { Extraída (AVE) }\end{array}$ \\
\hline Similaridade Percebida (SIM) & 0,962 & 0,962 & 0,806 \\
\hline Percepção de crowding (CROWD) & 0,954 & 0,955 & 0,875 \\
\hline Satisfação (SAT) & 0,968 & 0,968 & 0,911 \\
\hline
\end{tabular}

Fonte: Elaborada pelo pesquisador (2018).

O Cronbach's Alpha é um coeficiente que varia de 0 a 1 , e a literatura sugere que valores acima de 0,7 sejam considerados satisfatórios. Quanto à confiabilidade composta, as medidas foram calculadas para cada construto, a partir das cargas padronizadas e dos erros de mensuração das variáveis. O valor mínimo aceitável para a confiabilidade composta é 0,70 (Hair et al., 2005; Malhotra, 2006), o que também sinalizou a consistência interna dos indicadores que compõem o modelo.

Os indicadores também se mostraram adequados, especialmente quando se observa a variância média extraída (AVE). O valor mínimo considerado para a AVE é 0,50 (Hair et al., 2005; Kline, 2011). A validade discriminante foi realizada comparando-se a variância compartilhada entre cada par dos construtos (coeficiente de determinação) com a variância média extraída de cada construto (Fornell \& Larcker, 1981). 
Tabela 2. Correlações, Variância Compartilhada e AVE

\begin{tabular}{lccc}
\hline \multicolumn{1}{c}{ Variáveis } & $\begin{array}{c}\text { Similaridade } \\
\text { Percebida }\end{array}$ & $\begin{array}{c}\text { Percepção de } \\
\text { Crowding }\end{array}$ & Satisfação \\
\hline Similaridade Percebida &, 806 &,$- 036^{\star}$ &, $584^{\star \star}$ \\
\hline Percepção de Crowding &, 130 &, 875 &,$- 123^{\star \star}$ \\
\hline Satisfação &, 341 &,- 015 &, 911 \\
\hline
\end{tabular}

Fonte: Elaborada pelo pesquisador (2018).

Nota: os valores na diagonal mostram a AVE de cada construto; os valores acima da diagonal mostram os coeficientes de correlação entre os construtos; os valores abaixo da diagonal apresentam a variância compartilhada.

Analisando-se a Tabela 2, os valores na diagonal mostram a AVE (Variância Média Extraída) de cada construto e percebe-se que os valores da variância compartilhada não são superiores à variância média extraída dos construtos, indicando validade discriminante.

\section{Validação das condições do experimento}

Para averiguar a qualidade do experimento conduzido, as condições do desenho fatorial foram analisadas, ou seja, os níveis de densidade humana e proximidade física, bem como os perfis dos outros consumidores. Os resultados demonstraram que o cenário lido pelos participantes descreveu uma situação real de compra. A média dos respondentes foi de 5,26 (tendo 7 indicando concordo totalmente). Os participantes também concordaram com a afirmativa de que a imagem (fotografia do cenário), fornecida para ilustrar o contexto do ambiente varejista, representou uma situação real (média de 5,27). Os sujeitos de pesquisa também sinalizaram positivamente quanto ao questionamento sobre a facilidade de se imaginarem no cenário descrito no instrumento de coleta (média de 5,18). Assim, a validade interna quanto ao desenho experimental delineado foi atendida.

\section{Achados empíricos}

Inicialmente foi verificada a hipótese $\mathrm{H} 1$, cuja afirmação revela que consumidores distantes fisicamente da presença social no ambiente varejista percebem menos crowding em comparação com aqueles fisicamente próximos.

Tabela 3. Diferença na percepção de crowding conforme nível de proximidade física

\begin{tabular}{ccc|ccc}
\hline & \multicolumn{4}{c|}{ Distante Fisicamente $(\mathrm{n}=178)$} & \multicolumn{3}{c}{ Próximo Fisicamente $(\mathrm{n}=180)$} \\
\cline { 2 - 6 } & Média & DP & Média & DP & $\boldsymbol{p}$ \\
\hline Percepção de Crowding & 3,02 & 1,78 & 4,20 & 1,85 & $<0,001$ \\
\hline
\end{tabular}

Fonte: Elaborada pelo pesquisador (2018).

A média dos respondentes expostos à condição 'distante fisicamente' foi inferior e estatisticamente significativa quando comparada ao segundo grupo (próximo fisicamente), de modo que a H1 foi suportada. Logo, é 
coerente afirmar que mesmo sem haver interação social entre indivíduos que compartilham o ambiente varejista, a presença social ainda é capaz de influenciar respostas individuais (Argo et al., 2005; Xu, Shen, \& Wyer, 2012).

Esses achados consubstanciam o argumento de que a densidade humana, isoladamente, não explica totalmente a percepção de crowding. Além disso, sugere que a percepção de crowding e a relação com demais respostas do consumidor possam ocorrer em função de outras variáveis. Ao passo em que reitera resultados de estudos anteriores, o teste da hipótese H1 também permite que novos pressupostos sejam elaborados a fim de compreender em quais circunstâncias e contextos de consumo a densidade humana exercerá menos impacto sobre o consumidor.

Hui, Bradlow e Fader (2009) evidenciaram que a presença de outros clientes no ambiente de loja atrai consumidores para o estabelecimento, mesmo que o espaço físico apresente níveis altos de crowding. Portanto, infere-se que além da densidade humana e da proximidade física, as características observáveis dos outros consumidores (ex.: idade, gênero e aparência em geral) também precisam ser consideradas (Thakor, Suri, \& Saleh 2008). A hipótese H2a estabelece que a similaridade percebida modera o impacto da densidade humana sobre a percepção de crowding.

Tabela 4. Resultado do teste da hipótese $\mathrm{H} 2 \mathrm{a}$

\begin{tabular}{lcccc}
\hline \multicolumn{1}{c}{ Variável } & $\boldsymbol{\beta}$ & S.E & T & Sig. \\
\hline Constante & 3,614 & 0,078 & 46,192 & 0,001 \\
Similaridade Percebida & $-0,030$ & 0,045 & $-0,659$ & 0,070 \\
Densidade Humana & 2,380 & 0,155 & 15,212 & 0,001 \\
Interação & $-0,299$ & 0,091 & $-3,292$ & 0,001 \\
\hline
\end{tabular}

Fonte: Elaborada pelo pesquisador (2018).

Uma variável é considerada moderadora (M) em situações nas quais o relacionamento entre duas variáveis (ex.: densidade humana e percepção de crowding) ocorre em função de uma terceira (ex.: similaridade percebida) (Hayes, 2013). A significância dos coeficientes padronizados da regressão $\beta$ foi avaliada com interesse no valor da interação. Apesar do efeito condicional (isolado) da similaridade percebida ter se mostrado marginalmente significativo $(p=0,07)$, este impacta negativamente a percepção de crowding $(\beta=-0,03)$. Logo, quanto maior o nível de similaridade percebida, menor será a percepção de densidade humana por parte do consumidor.

Em relação à densidade humana, seu efeito se mostrou conforme o esperado, possuindo uma relação positiva $(\beta=2,38)$ com a variável dependente e estatisticamente diferente de zero ( $p \leq 0,001)$. Inclusive, a densidade é responsável pela maior parte da variação da percepção de crowding ( $R^{2}$ ajustado $\left.=42,69 \%\right)$. Observa-se que o efeito moderador da similaridade percebida na relação entre densidade humana e percepção de crowding se confirmou, posto que a interação entre variável dependente e moderadora sobre a dependente foi significativa $(\beta=-0,29$ e $p \leq 0,001)$.

Considerando que as pessoas estão mais sujeitas a favorecer e serem atraídas por coisas semelhantes (ex.: pessoas e objetos), a relação entre comportamento do consumidor e o ambiente varejista é afetada pela 
similaridade percebida do cliente-alvo para com os outros consumidores (Söderlund, 2011). A contextualização dos fatores sociais e a análise de seu efeito combinado sobre o comportamento do consumidor no ambiente varejista se mostram como uma alternativa válida à compreensão acerca do impacto da dimensão social da loja.

A percepção de crowding refere-se à dimensão subjetiva e à consequência emocional em função da densidade (Stokols, 1972). Mehta (2013) pondera que, mesmo em situações de alta densidade humana, ainda existe a possibilidade de serem observadas respostas positivas, desde que o crowding não seja percebido como um problema para o consumidor. O tipo de pessoas ou clientela que compõe o ambiente varejista pode determinar como o consumidor responderá ao crowding (Baker \& Wakefield, 2012).

A verificação da hipótese $\mathrm{H} 2 \mathrm{a}$ traz novas evidências que reiteram o argumento no sentido de compreender o fenômeno crowding enquanto um fenômeno multifacetado e dinâmico. Logicamente que a densidade humana corresponde ao antecedente direto da percepção de crowding, porém este refere-se à uma interpretação de caráter subjetivo do indivíduo, que considera outras pistas sociais ao avaliar a aglomeração humana. Neste sentido, a similaridade corresponde à variável que interfere no efeito da densidade humana. Desse modo, introduz empiricamente esta variável no estudo do comportamento do consumidor ao investigar o fenômeno crowding.

Entende-se que um cliente pode reagir de forma diferente ao perceber outros consumidores semelhantes, em comparação àqueles em relação aos quais não compartilha a mesma identidade social. Percepções favoráveis acerca dos outros clientes influenciam positivamente as respostas afetivas do consumidor que analisa a presença social (Uhrich \& Benkenstein, 2012). Neste sentido, foi realizada a verificação da hipótese H2b.

Assim como na hipótese H2a, aplicou-se a análise de moderação via regressão linear, através do método dos mínimos quadrados. Nesse modelo, o fator densidade humana assume o papel de variável independente, a similaridade percebida moderadora e a satisfação enquanto variável dependente. O intuito foi verificar se a inclusão da variável similaridade percebida interfere na magnitude do impacto da percepção de crowding $(\beta)$ sobre a satisfação, ou seja, se seu efeito é reduzido.

Tabela 5. Modelos de regressão para a satisfação do consumidor enquanto variável dependente

\begin{tabular}{lccc}
\hline \multicolumn{1}{c}{ Modelo } & $\mathbf{R}$ & $\mathbf{R}^{\mathbf{2}}$ & $\mathbf{R}^{\mathbf{2}}$ ajustado \\
\hline Crowding & 0,123 & 0,015 & 0,012 \\
Crowding e Similaridade Percebida & 0,593 & 0,352 & 0,348 \\
\hline
\end{tabular}

Fonte: Elaborada pelo pesquisador (2018).

Ao analisar a relação entre crowding e satisfação, verificou-se que a similaridade percebida assume papel importante, sobretudo na minimização do impacto negativo que a densidade humana possui sobre as respostas do consumidor. A seguir tem-se os coeficientes padronizados com seus respectivos níveis de significância para cada modelo. 
Tabela 6. Coeficientes de regressão conforme inclusão da similaridade percebida no modelo

\begin{tabular}{llcccc}
\hline & Variáveis & $\beta$ & S.E & T & Sig. \\
\hline Modelo 1 & Crowding & $-0,101$ & 0,042 & $-1,907$ & 0,057 \\
& Constante & 4,031 & 0,171 & 23,520 & 0,001 \\
Modelo 2 & & & & \\
& $\quad$ Crowding &,- 078 & 0,033 & $-1,895$ & 0,059 \\
& Similaridade Percebida &, 627 & 0,037 & 15,270 & 0,001 \\
& Constante & 1,799 & 0,198 & 9,090 & 0,001 \\
\hline
\end{tabular}

Fonte: Elaborada pelo pesquisador (2018).

Analisando a Tabela 6, fica evidente que, no modelo de regressão em que a similaridade percebida está inserida, a influência negativa da percepção de crowding sobre a satisfação $(\beta=-0,08)$ diminui em comparação ao modelo $1(\beta=-0,10)$. Além disso, as relações entre as três variáveis se mostraram estatisticamente significantes ( $p \leq 0,001)$. Esses resultados se alinham aos achados provenientes do estudo de Raajpoot, Jackson e Lefebvre (2013), os quais confirmaram a hipótese de que a similaridade percebida interfere na satisfação do consumidor.

As variações de densidade humana nas lojas afetam a percepção de crowding e a satisfação dos consumidores (Brandão \& Parente, 2012). Os clientes, como parte do ambiente de consumo, afetam uns aos outros (direta ou indiretamente) durante a experiência de compra (Baker, 1986; Martin \& Pranter, 1989; Bitner, 1992; Martin, 1996; Baker et al., 2002; Moore et al., 2005).

Em relação à similaridade percebida, seu efeito ocorre a partir do processamento de informação, ou seja, uma pessoa utiliza as informações referentes à(s) outra(s) para formar sua própria impressão acerca da outra. Os resultados se alinham aos achados oriundos de estudos anteriores que investigaram o papel da similaridade percebida enquanto antecedente das respostas do consumidor no ambiente varejista (Argo et al., 2008; Borges et al., 2010; Kwon, Ha, \& Im, 2016).

Diante do exposto, esses achados fornecem um claro entendimento de como a presença de outros clientes pode influenciar as avaliações individuais acerca do varejista e a própria experiência de compra. O construto satisfação corresponde a uma variável utilizada por acadêmicos e praticantes da área para compreender os processos cognitivos e afetivos que levam o consumidor a ajuizar acerca de bens, serviços, ambiente e o próprio varejista. Ao testar a hipótese $\mathrm{H} 2 \mathrm{a}$, o trabalho avança no sentido de fornecer mais elementos a serem considerados ao investigar o fenômeno crowding.

\section{Conclusões}

O presente estudo investigou de que modo a similaridade percebida modera a relação entre crowding e respostas do consumidor. Os resultados evidenciam que a similaridade percebida mitiga o impacto da densidade humana sobre a percepção de crowding, assim como o efeito da percepção de crowding sobre a satisfação do consumidor. O estudo traz uma contribuição 
relevante para o campo de pesquisas envolvidas no entendimento dos fatores sociais que interferem nas respostas individuais em ambientes varejistas.

Além de introduzir uma nova variável a fim de melhor apreender o fenômeno crowding, o estudo enfatiza que a similaridade percebida pode determinar o êxito do varejista quanto à experiência de consumo. Por este motivo que cuidados quanto à disponibilidade de espaço e atendentes, monitoramento do fluxo de clientes e até mesmo a realização de transações comerciais pela Internet podem ajudar os gerentes de loja receber adequadamente os clientes em seus estabelecimentos.

Ao fazer uso da Teoria do Impacto Social (Latané, 1981) e do Paradigma Similaridade Atração (Byrne, 1971) enquanto base teórica, o estudo reforça a relevância destas duas abordagens para o estudo do comportamento do consumidor. Em outras palavras, amplia-se as possibilidades de investigação da dimensão social de ambientes varejistas a partir dessas duas teorias, que se mostraram pertinentes e relevantes quanto à explicação do fenômeno aqui investigado.

Complementarmente, ao considerar efeitos moderadores na relação entre variáveis, o trabalho contribui para que sejam observados fenômenos e contextos no campo de estudo do comportamento do consumidor, considerando variáveis que podem interferir na dinâmica de associações teóricas previamente estabelecidas na literatura. Isso permite que novas variáveis sejam inseridas em modelos consolidados, permitindo assim uma atualização destes e, por conseguinte, um avanço no conhecimento na área.

Muito embora o varejo contemporâneo direcione seus esforços no gerenciamento de grandes volumes de dados (Big Data) e na aplicação de Business e Retail Analytics, este artigo ressalta que o ambiente físico ainda possui sua pertinência. Neste sentido, sugere-se investigar daqui em diante a integração entre as duas dimensões do varejo (off-line e online), denominado Omni-channel Retailing (Verhoef, Kannan, \& Inman, 2015).

\section{Nota}

Os autores agradecem os avaliadores pelas as contribuições para o aprimoramento do artigo, por meio de recomendações e sugestões de melhoria.

\section{Referências}

Aaker, D. A., Kumar, V., \& Day, G. S. (2001). Pesquisa de marketing. São Paulo: Atlas.

Aguiar, E. C., \& Farias, S. A.. (2015). Percepção da presença dos outros consumidores e sua relação com emoções e valor hedônico de compra. RAE - Revista de Administração de Empresas, 55(6), 712-723.

Aguiar, E. C., Farias, S. A., Gomes, V. M. S., \& Santos, J. G. (2015). Percepção de crowding e comportamento do consumidor: Uma abordagem não linear no varejo supermercadista de baixa renda. Revista de Administração da UNIMEP, 13(3), 207-229. 
Alnabulsi, H., \& Drury, J. (2014). Social identification moderates the effect of crowd density on safety at the Hajj. Proceedings of the National Academy of Sciences, 111(25), 9091-9096.

Argo, J. J., Dahl, D. W., \& Manchanda, R. V. (2005). The influence of a mere social presence in a retail context. Journal of Consumer Research, 32(2), 207-212.

Argo, J. J., Dahl, D. W., \& Morales, A. C. (2008). Positive consumer contagion: Responses to attractive others in a retail context. Journal of Marketing Research, 45(6), 690-701.

Baker, J. (1986). The Role of the Environment in Marketing Services: The Consumer Perspective. In J. A. Czepiel, C. A. Congram, \& J. Shanahan (Orgs.). The Services Challenge: Integrating for Competitive Advantage (pp.79-84). Chicago: American Marketing Association.

Baker, J., Parasuraman, A., Grewal, D., \& Voss, G. B. (2002). The influence of multiple store environment cues on perceived merchandise value and patronage intentions. Journal of marketing, 66(2), 120-141.

Baker, J., \& Wakefield, K. L. (2012). How consumer shopping orientation influences perceived crowding, excitement, and stress at the mall. Journal of the Academy of Marketing Science, 40(6), 791-806.

Baron, R. M., \& Kenny, D. A. (1986). The moderator-mediator variable distinction in social psychological research: Conceptual, strategic, and statistical considerations. Journal of Personality and Social Psychology, 51(6), 1173-1182.

Bearden, W. O., \& Etzel, M. J. (1982). Reference group influence on product and brand purchase decisions. Journal of Consumer Research, 9(2), 183-194.

Bitner, M. J. (1992). Servicescapes: The impact of physical surroundings on customers and employees. The Journal of Marketing, 56(2), 57-71.

Blau, G. J., \& Katerberg, R. Toward enhancing research with the social information processing approach to job design. Academy of Management Review, 7(4), 543550.

Borges, A., Chebat, J. C., \& Babin, B. J. (2010). Does a companion always enhance the shopping experience?. Journal of Retailing and Consumer Services, 17(4), 294299.

Brandão, M. M., Parente, J., \& Oliveira, B. B. (2010). Percepção de crowding no varejo: uma investigação exploratória no mercado Brasileiro. RAE-eletrônica, 9(2).

Brandão, M. M. (2011). Crowding no varejo: diferenças na satisfação e percepção de valor hedônico de consumidores de alta e baixa renda no Brasil. Tese de doutorado, Escola de Administração de Empresas de São Paulo - FGV/EAESP, São Paulo, SP, Brasil.

Brandão, M. M., \& Parente, J. (2012). Brasileiro gosta de "muvuca"? Impacto da densidade humana no comportamento de compra. Revista de Administração de Empresas - RAE, 52(6), 613-627.

Brocato, E. D., Voorhees, C. M., \& Baker, J. (2012). Understanding the influence of cues from other customers in the service experience: A scale development and validation. Journal of retailing, 88(3), 384-398.

Byrne, D. (1971). The attraction paradigm. New York: Academic Press. 
Campbell, D. T., \& Stanley, J. C. (1979). Delineamentos experimentais e quase-experimentais de pesquisa. São Paulo: EPU-EDUSP.

Cronbach, L. J. (1951). Coefficient alpha and the internal structure of tests. Psychometrika, 16(3), 297-334.

Emiley, S. F. (1975). The effects of crowding and interpersonal attraction on affective responses, task performance, and verbal behavior. The Journal of Social Psychology, 97(2), 267-278.

Eroglu, S. A., \& Harrell, G. D. (1986). Retail crowding: Theoretical and strategic implications. Journal of retailing, 62(4), 346-363.

Evans, K. R., Christiansen, T., \& Gill, J. D. (1996). The impact of social influence and role expectations on shopping center patronage intentions. Journal of the Academy of Marketing Science, 24(3), 208-218.

Fisher, J. D., \& Byrne, D. (1975). Too close for comfort: Sex differences in response to invasions of personal space. Journal of Personality and Social Psychology, 32(1), 15-21

Flick, U. (2009). Uma introdução à pesquisa qualitativa (2nd ed.). Porto Alegre: Bookman.

Fornell, C., \& Larcker, D. F. (1981). Evaluating structural equation models with unobservable variables and measurement error. Journal of marketing research, 18(1), 39-50.

Grove, S. J., \& Fisk, R. P. (1997). The impact of other customers on service experiences: a critical incident examination of "getting along". Journal of retailing, 73(1), 63-85.

Grush, J. E., Clore, G. L., \& Costin, F. (1975). Dissimilarity and attraction: when difference makes difference. Journal of Personality and Social Psychology, 32(3), 783-789.

Hair, J. F., Anderson, R. E., Tatham, R. L., \& Black, W. C. (2005). Análise multivariada de dados. (4th ed.). Englewood Cliffs: Prentice-Hall.

Harrell, G. D., \& Hutt, M. D. (1976). Buyer behavior under conditions of crowding: An initial framework. Advances in Consumer Research, 3(1), 36-39.

Harris, B., Luginbuhl, J. E., \& Fishbein, J. E. (1978). Density and personal space in a field setting. Social Psychology, 41(4), 350-353.

Hayes, A. F. (2013). Introduction to mediation, moderation, and conditional process analysis: A regression-based approach. New York: The Guilford Press.

Hernandez, J. M., Basso, K., \& Brandão, M. M. (2014). Pesquisa experimental em marketing. Revista Brasileira de Marketing - REMark, 13(2), 96-115.

Hui, M. K., \& Bateson, J. E. (1991). Perceived control and the effects of crowding and consumer choice on the service experience. Journal of consumer research, 18(2), 174-184.

Hui, S. K., Bradlow, E. T., \& Fader, P. S. (2009). Testing behavioral hypotheses using an integrated model of grocery store shopping path and purchase behavior. Journal of consumer research, 36(3), 478-493.

Jin, L., He, Y., \& Zhang, Y. (2014). How power states influence consumers' perceptions of price unfairness. Journal of Consumer Research, 4O(5), 818-833. 
Kim, N., \& Lee, M. (2012). Other customers in a service encounter: examining the effect in a restaurant setting. Journal of Services Marketing, 26(1), 27-40.

Kline, R. B. (2011). Principles and practice of structural equation modeling. (3rd ed.). New York: The Guilford Press.

Kraut, R. E. (1982). Social presence, facial feedback, and emotion. Journal of Personality and Social Psychology, 42(5), 853-863.

Kwon, H., Ha, S., \& Im, H. (2016). The impact of perceived similarity to other customers on shopping mall satisfaction. Journal of Retailing and Consumer Services, 28(1), 304-309.

Latané, B. (1981). The psychology of social impact. American Psychologist, 36(4), 343-356.

Lee, S. Y., Kim, J. O., \& Li, J. G. (2011). Impacts of store crowding on shopping behavior and store image. Journal of Asian Architecture and Building Engineering, 10(1), 133140.

Loo, C. (1975). The psychological study of crowding. The American Behavioral Scientist, 18(6), 826-443.

Machleit, K. A., Eroglu, S. A., \& Mantel, S. P. (2000). Perceived retail crowding and shopping satisfaction: what modifies this relationship?. Journal of consumer psychology, 9(1), 29-42.

Mackinnon, S. P., Jordan, C. H., \& Wilson, A. E. (2011). Birds of a feather sit together: Physical similarity predicts seating choice. Personality and Social Psychology Bulletin, 37(7), 879-892.

Maeng, A., Tanner, R. J., \& Soman, D. (2013). Conservative when crowded: Social crowding and consumer choice. Journal of Marketing Research, 50(6), 739-752.

Malhotra, N, K. (2006). Pesquisa de marketing: uma orientação aplicada. (4th ed.). São Paulo: Prentice Hall.

Martin, C. L. (1996). Consumer to consumer relationships: satisfaction with other consumers' public behavior. Journal of Consumer Affairs, 30(1), 146-169.

Martin, C. L., \& Pranter, C. A. (1989). Compatibility management: customer-tocustomer relationships in service environments. Journal of Services Marketing, 3(3), 5-15.

McFerran, B., \& Argo, J. J. (2014). The entourage effect. Journal of Consumer Research, 4O(5), 871-884.

McGrath, M. A., \& Otnes, C. (1995). Unacquainted influencers: when strangers interact in the retail setting. Journal of Business Research, 32(3), 261-272.

Mehta, R. (2013). Understanding perceived retail crowding: A critical review and research agenda. Journal of Retailing and Consumer Services, 20(6), 642-649.

Mehta, R., Sharma, N. K., \& Swami, S. (2013). The impact of perceived crowding on consumers' store patronage intentions: Role of optimal stimulation level and shopping motivation. Journal of Marketing Management, 28(8), 812-835.

Miao, L., Mattila, A. S., \& Mount, D. (2011). Other consumers in service encounters: a script theoretical perspective. International Journal of Hospitality Management, 30(4), 933-941. 
Michon, R., Chebat, J. C., \& Turley, L. W. (2005). Mall atmospherics: the interaction effects of the mall environment on shopping behavior. Journal of Business Research, 58(1), 576-583.

Montoya, R. M., Horton, R. S., \& Kirchner, J. (2008). Is actual similarity necessary for attraction? A meta-analysis of actual and perceived similarity. Journal of Social and Personal Relationships, 25(6), 889-922.

Montoya, R. M., \& Horton, R. S. (2012). A meta-analytic investigation of the processes underlying the similarity-attraction effect. Journal of Social and Personal Relationships, 30(1), 64-94.

Moore, R., Moore, M. L., \& Capella, M. (2005). The impact of customer-tocustomer interactions in a high personal contact service setting. Journal of Services Marketing, 19(7), 482-491.

Pan, Y., \& Siemens, J. C. (2011). The differential effects of retail density: An investigation of goods versus service settings. Journal of Business Research, 64(1), 105-112.

Preacher, K. J., \& Hayes, A. F. (2008). Asymptotic and resampling strategies for assessing and comparing indirect effects in multiple mediator models. Behavior research methods, 40(3), 879-891.

Preacher, K. J., Rucker, D. D., \& Hayes, A. F. (2007). Addressing moderated mediation hypotheses: Theory, methods, and prescriptions. Multivariate behavioral research, 42(1), 185-227.

Quezado, I., Costa, J. S., Peñaloza, V., Matos, F. R. N., \& Ferraz, S. B. (2016). Sempre cabe mais um? Uma investigação cross-cultural do comportamento do consumidor em ambiente aglomerado. Revista de Administração FACES Journal, 14(3), 56-74.

Raajpoot, N., Jackson, A., \& Lefebvre, J. (2013). Non-verbal customer-to-customer interaction in retail setting: An investigation of indirect effects of perceived customer similarity on important marketing outcomes. Atlantic Marketing Journal, 2(1), 16-41.

Rüstemli, A. (1991). Crowding effects of density and interpersonal distance. The Journal of social psychology, 132(1), 51-58.

Ryan, M. J. (1982). Behavioral intention formation: The interdependency of attitudinal and social influence variables. Journal of Consumer Research, 9(3), 263-278.

Söderlund, M. (2011). Other customers in the retail environment and their impact on the customer's evaluations of the retailer. Journal of Retailing and Consumer Services, 18(3), 174-182.

Stokols, D. (1972). On the distinction between density and crowding: Some implications for future research. Psychological review, 79(3), 275-278.

Tabachnick, B. G., \& Fidell, L. S. (2013). Using multivariate statistics (6th ed.). Boston: Pearson.

Thakor, M. V., Suri, R., \& Saleh, K. (2008). Effects of service setting and other consumers' age on the service perceptions of young consumers. Journal of Retailing, 84(2), 137-149.

Tombs, A. G., \& McColl-Kennedy, J. R. (2010). Social and spatial influence of customers on other customers in the social-servicescape. Australasian Marketing Journal - AMJ, 18(3), 120-131. 
Uhrich, S., \& Benkenstein, M. (2012). Physical and social atmospheric effects in hedonic service consumption: customers' roles at sporting events. The Service Industries Journal, 32(1), 1741-1757.

Uhrich, S., \& Luck, M. (2012). Not too many but also not too few: Exploring the explanatory mechanisms for the negative effects of low customer density in retail settings. Qualitative Market Research: An International Journal, 15(3), 290-308.

Verhoef, P. C., Kannan, P. K., \& Inman, J. J. (2015). From multi-channel retailing to omni-channel retailing: introduction to the special issue on multi-channel retailing. Journal of retailing, 91(2), 174-181.

Vieira, V. A. (2009). Moderação, mediação, moderadora-mediadora e efeitos indiretos em modelagem de equações estruturais: uma aplicação no modelo de desconfirmação de expectativas. Revista de Administração da Universidade de São Paulo - RAUSP, 44(1), 17-33.

White,R.W.(1959). Motivationreconsidered:Theconceptofcompetence.Psychological review, 66(5), 297-333.

Wu, L. L., Mattila, A. S., \& Han, J. R. (2014). Territoriality revisited: Other customer's perspective. International Journal of Hospitality Management, 38(1), 48-56.

Xu, J., Shen, H., \& Wyer, R. S., Jr. (2012). Does the distance between us matter? Influences of physical proximity to others on consumer choice. Journal of Consumer Psychology, 22(3), 418-423.

Yüksel, A. (2009). Exterior color and perceived retail crowding: Effects on tourists' shopping quality inferences and approach behaviors. Journal of Quality Assurance in Hospitality \& Tourism, 10(4), 233-254.

Zhang, X., Li, S., Burke, R. R., \& Leykin, A. (2014). An examination of social influence on shopper behavior using video tracking data. Journal of Marketing, 78(9), 24-41. 